\title{
Isotemporal substitution of inactive time with physical activity and time in bed: cross-sectional associations with cardiometabolic health in the PREDIMED- Plus study
}

Aina M. Galmes-Panades ${ }^{1,2}$, Veronica Varela-Mato33, Jadwiga Konieczna ${ }^{1,2}$, Julia Wärnberg ${ }^{1,4}$, Miguel Ángel Martínez-González ${ }^{1,5,6}$, Jordi Salas-Salvadó ${ }^{1,7,8}$, Dolores Corella ${ }^{1,9}$, Helmut Schröder ${ }^{10,11}$, Jesús Vioque ${ }^{11,12}$, Ángel M. Alonso-Gómez 1,13, J. Alfredo Martínez ${ }^{1,14,15}$, Luís Serra-Majem ${ }^{1,16}$, Ramon Estruch ${ }^{1,17}$, Francisco J. Tinahones ${ }^{1,18}$, José Lapetra ${ }^{1,19}$, Xavier Pintó 1,20,21, Josep A. Tur ${ }^{1,2,22}$, Antonio Garcia-Rios ${ }^{1,23}$, Blanca Riquelme-Gallego ${ }^{11,24}$, José Juan Gaforio ${ }^{11,25}$, Pilar Matía-Martín ${ }^{26}$, Lidia Daimiel ${ }^{27}$, Rafael Manuel Micó Pérez ${ }^{28}$, Josep Vidal ${ }^{29,30}$, Clotilde Vázquez ${ }^{1,31}$, Emilio Ros ${ }^{1,32}$, Ana Garcia-Arellano ${ }^{1,5,33}$, Andrés Díaz-López ${ }^{1,7,8}$, Eva M. Asensio ${ }^{1,9}$, Olga Castañer ${ }^{1,10}$, Francisca Fiol ${ }^{34}$, Luis Alfredo Mira-Castejón ${ }^{35}$, Anai Moreno Rodríguez ${ }^{1,13}$, Juan Carlos Benavente- Marín ${ }^{4}$, Itziar Abete ${ }^{1,14}$, Laura Tomaino 1,16,36 ${ }^{\text {, Rosa Casas }}{ }^{1,17}$, F. Javier Barón López ${ }^{4}$, José Carlos Fernández-García ${ }^{1,18}$, José Manuel Santos-Lozano ${ }^{1,19}$, Ana Galera 1,20, Catalina M. Mascaró ${ }^{1,2,22}$, Cristina Razquin ${ }^{1,5}$, Christopher Papandreou ${ }^{1,7,8}$, Olga Portoles ${ }^{1,9}$, Karla Alejandra Pérez-Vega ${ }^{1,10}$, Miguel Fiol ${ }^{1,2}$, Laura Compañ-Gabucio ${ }^{12}$, Jessica Vaquero-Luna ${ }^{1,13}$, Miguel Ruiz-Canela ${ }^{1,5}$, Nerea Becerra-Tomás ${ }^{1,7,8}$, Montserrat Fitó ${ }^{1,10}$ and Dora Romaguera ${ }^{1,2^{*}}$

\section{Abstract}

Background: This study explored the association between inactive time and measures of adiposity, clinical parameters, obesity, type 2 diabetes and metabolic syndrome components. It further examined the impact of reallocating inactive time to time in bed, light physical activity (LPA) or moderate-to-vigorous physical activity (MVPA) on cardio-metabolic risk factors, including measures of adiposity and body composition, biochemical parameters and blood pressure in older adults.

Methods: This is a cross-sectional analysis of baseline data from 2189 Caucasian men and women (age 55-75 years, BMl $27-40 \mathrm{Kg} / \mathrm{m}^{2}$ ) from the PREDIMED-Plus study (http://www.predimedplus.com/). All participants had $\geq 3$ components of the metabolic syndrome. Inactive time, physical activity and time in bed were objectively determined using triaxial accelerometers GENEActiv during 7 days (ActivInsights Ltd., Kimbolton, United Kingdom). Multiple adjusted linear and logistic regression models were used. Isotemporal substitution regression modelling was performed to assess the (Continued on next page)

\footnotetext{
* Correspondence: mariaadoracion.romaguera@ssib.es

${ }^{1}$ Centro de Investigación Biomédica en Red Fisiopatología de la Obesidad y

la Nutrición (CIBEROBN), Institute of Health Carlos III, Madrid, Spain

${ }^{2}$ Research Group on Nutritional Epidemiology \& Cardiovascular

Physiopathology. Health Research Institute of the Balearic Islands (IdISBa),

University Hospital Son Espases, Balearic Islands, Spain

Full list of author information is available at the end of the article
}

(c) The Author(s). 2019 Open Access This article is distributed under the terms of the Creative Commons Attribution 4.0 International License (http://creativecommons.org/licenses/by/4.0/), which permits unrestricted use, distribution, and reproduction in any medium, provided you give appropriate credit to the original author(s) and the source, provide a link to the Creative Commons license, and indicate if changes were made. The Creative Commons Public Domain Dedication waiver (http://creativecommons.org/publicdomain/zero/1.0/) applies to the data made available in this article, unless otherwise stated. 
(Continued from previous page)

relationship of replacing the amount of time spent in one activity for another, on each outcome, including measures of adiposity and body composition, biochemical parameters and blood pressure in older adults.

Results: Inactive time was associated with indicators of obesity and the metabolic syndrome. Reallocating 30 min per day of inactive time to 30 min per day of time in bed was associated with lower BMl, waist circumference and glycated hemoglobin $(\mathrm{HbA} 1 \mathrm{c}$ ) (all p-values < 0.05). Reallocating 30 min per day of inactive time with 30 min per day of LPA or MVPA was associated with lower BMI, waist circumference, total fat, visceral adipose tissue, HbA1c, glucose, triglycerides, and higher body muscle mass and HDL cholesterol (all p-values < 0.05).

Conclusions: Inactive time was associated with a poor cardio-metabolic profile. Isotemporal substitution of inactive time with MVPA and LPA or time in bed could have beneficial impact on cardio-metabolic health.

Trial registration: The trial was registered at the International Standard Randomized Controlled Trial (ISRCTN: http://www. isrctn.com/ISRCTN89898870) with number 89898870 and registration date of 24 July 2014, retrospectively registered.

Keywords: Inactive time, Light physical activity, Moderate to vigorous physical activity, Time in bed, Cardiometabolic risk, Isotemporal substitution

\section{Background}

Cardio-metabolic diseases such as type 2 diabetes (T2D) and the metabolic syndrome (MetS) are increasingly prevalent worldwide [1, 2]. Overweight and obesity are major risk factors for these metabolic alterations [3-5] and the World Health Organization (WHO) has projected a significant increase by 2030 [1, 3, 6, 7]. A vast body of the literature suggests that physical activity (PA) and sedentary behaviours, including inactive time and time in bed, are strongly and independently associated with markers of obesity, body composition, and the MetS [2, 8-20]. However, current public health guidelines are mostly focused on the health benefits of moderate-to-vigorous physical activity (MVPA) and less attention is given to inactive time [21, 22].

Research in older adults highlights the health benefits of MVPA and light physical activity (LPA) [2, 4, 23, 24]. However, limited research has explored the associations between time spent inactive, time in bed and cardiometabolic health in an aging population [4, 13, 23, 25-28]; and, limited research has explored these associations attending the 24-h finite time of a day in a population with chronic conditions.

Isotemporal substitution models have been recommended as one of the most appropriate statistical analysis to explore the associations between reallocating activity patterns, particularly time spent inactive, and health outcomes $[4,13,23$, 26, 28-30]. This type of analyses takes into account that time is finite. Thus, spending time in one behavior (ie. Inactive time) results in less time being spent in another (ie. MVPA) [29], and understands that daily behaviours (sleep, sedentarism and physical activity) are co-dependent [31]. This will provide insightful information that will help better understand the impact of reallocating activity patterns in cardio-metabolic markers in older adults. This is crucial for the design of effective tailored interventions to improve cardiometabolic health in older people in the future. Therefore, this novel study aims to provide new evidence about the associations of inactive time with cardio-metabolic risk factors in an aging population. The outcomes were markers of cardiometabolic health: measures of adiposity and body composition, biochemical parameters, blood pressure, obesity, type 2 diabetes and metabolic syndrome components. The objectives of the present study were a) to explore crosssectional associations between inactive time and cardiometabolic risk factors; and b) to assess the impact of replacing $30 \mathrm{~min}$ per day of inactive time by $30 \mathrm{~min}$ of LPA, MVPA and time in bed on markers of cardio-metabolic health.

\section{Material and methods}

\section{Study overview and sample}

The PREDIMED-Plus study is a 6-year ongoing multicenter, randomized clinical trial, with two intervention arms for the primary prevention of cardiovascular disease in Spain. Details of the study's protocol have been described elsewhere [32] and are available on the website http://www.predimedplus.com/. Briefly, participant's in the intervention are receive a multicomponent weight loss intervention that includes an energy-restricted traditional Mediterranean Diet (erMedDiet), PA promotion and behavioural support. Those in the control group receive information about the Mediterranean Diet and cardiovascular health guidelines only. The aim of the study is to prevent cardiovascular disease (a composite of cardiovascular death, non-fatal myocardial infarction, and non-fatal stroke). Eligible participants were men aged 55-75 and women aged 60-75 years, with body mass index (BMI) $\geq 27$ and $<40 \mathrm{~kg} / \mathrm{m}^{2}$, who met $\geq 3 \mathrm{com}$ ponents of the MetS [33]. Overall, 6874 men and women were recruited and randomized into the study between 2013 and 2016 across 23 Spanish centers distributed throughout the country's geography; a subsample of 
2260 participants wore an accelerometer at baseline. Participants were asked to wear the accelerometer continuously for at least 7 days. Of those days, we excluded invalid days, i.e. those with less of $10 \mathrm{~h}$ of data per day. In addition, we excluded participants with less than 3 days of data [34-36]. Therefore, 2189 participants had valid data, defined as 3 or more days of data with more than $10 \mathrm{~h}$ recorded each day. Out of 2189 participants with accelerometer, 662 had additional data on body composition obtained from Dual-energy X-ray absorptiometry (DXA) measurements. All participants provided written informed consent. The study's protocol was approved by the Research Ethic Committees from all recruiting centers according to the ethical standards of the Declaration of Helsinki. The trial was registered at the International Standard Randomized Controlled Trial (ISRCTN: http://www.isrctn.com/ISRCTN89898870).

\section{Exposure assessment}

Participants were asked to wear an accelerometer on their non-dominant wrist (GENEActiv, ActivInsights Ltd., Kimbolton, United Kingdom) continuously for 7 days. The GENEActiv is a triaxial accelerometer with a dynamic range of $\pm 8 g$, where $g$ is equal to the Earth's gravitational pull. The GENEActiv was set to capture and store accelerations at a sampling frequency of $40 \mathrm{~Hz}$ [37]. As these activity counts are time and date stamped, detailed data on the time, volume, and intensity of movements can be derived [38].

Wrist-worn 3-axial accelerometers do not permit to distinguish standing from sitting or reclining postures, a fact that has conditioned the use of the term inactivity (include all postures) instead of sedentarism (only sitting or reclining postures) in the current study.

Data extracted from the GENEActiv (all in bouts of at least $1 \mathrm{~min}$ ) was clustered as: inactive time (cut-off intensity level used was $<40 \mathrm{mg}$ ) for those behaviours during waking hours equivalent to $<1.5$ Metabolic Equivalent Task, METs; LPA (cut-off intensity level used was $\geq 40 \mathrm{mg}$ and $<100 \mathrm{mg}$ ) equivalent to 1.5-3 METs; MVPA (cut-off intensity level used was $\geq 100 \mathrm{mg}$ ) equivalent to $>3$ METs; and time in bed (time between going to bed and leaving, calculated using a validated heuristic algorithm from accelerometer raw data unaided by a sleep diary) $[37,39,40]$. For sensitivity analyses we used accelerometer estimated data on sleep time (calculated from accumulated sustained inactivity bouts (SIB) during time in bed, excluding short wake periods ( $\mathrm{min} / \mathrm{night})$. SIB are detected as the absence of change in arm angle greater than 5 degrees for 5 min or more [41]); nevertheless, our main models are based on time in bed data, given that this estimation has been validated when no information from sleep diaries is available.

Raw data files were managed on servers at the University of Malaga and processed with R-package (R Core Team,
Vienna, Austria) using the open-source R-package GGIR, version 1.2-5 (cran.rproject.org/web/packages/GGIR/index. $\mathrm{html}$ ). This open sources code has been validated in relation to self-calibrated functions [42].

\section{Outcome assessment \\ Obesity}

Obesity prevalence and obesity indicators were determined based on anthropometric parameters. Anthropometric variables were measured by trained personnel according to PREDIMED-Plus protocol [32]. Body weight $(\mathrm{kg})$ and height $(\mathrm{cm})$ were measured in light clothing and without shoes using calibrated scales and a wall-mounted stadiometer. BMI was calculated by dividing the weight $(\mathrm{kg})$ by height in meters squared $\left(\mathrm{m}^{2}\right)$. Obesity was defined as a BMI $\geq 30 \mathrm{~kg} /$ $\mathrm{m}^{2}$, and overweight as a BMI $\geq 27$ and $<30 \mathrm{~kg} / \mathrm{m}^{2}$ (given the inclusion criteria, all of our participants had a $\mathrm{BMI} \geq 27$ and $<40 \mathrm{~kg} / \mathrm{m}^{2}$ ). Waist circumference (WC) was measured at the middle point between the last rib and the iliac crest. All anthropometric variables were determined in duplicate, and the average of the two measurements was used.

\section{Body composition}

Baseline data on total and regional body composition was measured using two types of DXA equipment belonging to the third-generation scanners from GE Healthcare, Madison - WI, connected with EnCore ${ }^{\mathrm{Tx}}$ software, depending on the availability of this material in the recruiting centers. Total body fat mass (expressed as percentage of total body mass), total body muscle mass (expressed as percentage of total body mass) and Visceral Adipose Tissue (VAT) mass (in $\mathrm{kg}$ ) were measured. For VAT measures, scans were reanalyzed using validated CoreScan software application [43]. These algorithms work through detection of the width of the subcutaneous tissue layer on the lateral part of the abdomen and the anterior-posterior thickness of the abdomen, by X-ray attenuation of the abdominal cavity in the android region. DXA scans were performed by trained operators following standard protocol and subject positioning provided by the manufacturer. The DXA was phantom calibrated daily according to manufacturer guidelines.

\section{Biochemical analyses and clinical determinations}

Blood samples were collected after $12 \mathrm{~h}$ of overnight fast and biochemical analysis were performed on fasting plasma to determine glucose, glycated hemoglobin (HbA1c), lowdensity (LDL)-cholesterol, high-density lipoprotein (HDL)cholesterol and triglycerides concentrations using enzymatic methods. Blood pressure was measured three times with a validated semiautomatic oscillometer (Omron HEM-705CP, the Netherlands) at 5, 10 and $15 \mathrm{~min}$ of rest whilst in a seated position. 


\section{Metabolic syndrome}

MetS was defined according to the International Diabetes Federation and the American Heart Association and National Heart, Lung and Blood Institute [33], as having at least 3 of the following components: abdominal obesity for European individuals (WC $\geq 88 \mathrm{~cm}$ in women and $\geq 102 \mathrm{~cm}$ in men), hypertriglyceridemia $(\geq 150 \mathrm{mg} / \mathrm{dL})$ or drug treatment for high plasma triglyceride concentration, low HDL $(<50 \mathrm{mg} / \mathrm{dL}$ in women and $<40 \mathrm{mg} / \mathrm{dL}$ in men), high blood pressure (systolic blood pressure (SBP) $\geq 130 \mathrm{mmHg}$ or diastolic blood pressure (DBP) $\geq 85 \mathrm{mmHg}$ ) or antihypertensive drug treatment, or high fasting glucose $(\geq 100 \mathrm{mg} / \mathrm{dL})$ or drug treatment for T2D. The presence of MetS was part of the inclusions criteria.

\section{Type 2 diabetes}

T2D was defined as meeting any of the following criteria: self-reported diabetes at inclusion or baseline, $\mathrm{HbA} 1 \mathrm{c} \geq 6.5 \%$ or use of antidiabetic medication at baseline, such as insulin, metformin (in case of diagnosed diabetes or $\mathrm{Hba} 1 \mathrm{c} \geq 6.5 \%$ ), and other medication for diabetes.

\section{Covariate assessment}

Baseline data on sex, age, smoking habits, educational level, erMedDiet, marital status, medical conditions and medication use have been evaluated using self-reported questionnaires. Smoking habits was categorized as current, former and never smoker; educational level was categorized as higher education/technician, secondary education and notcompleted primary education/primary education; marital status was categorized as married and not married, which included single/separated/divorced/widow (er). Adherence to an energy-restricted Mediterranean diet was measured using a 17-items ErMedDiet score (score range, 0-17; higher scores indicate greater adherence). This score is a modified version of the validated 14-item MEDAS (Mediterranean diet Adherence Screener) used in the PREDIMED study [44]. We also used data on objectively measured muscle strength. Lower-limb muscle strength was determined at baseline using previously validated in community-dwelling older subjects 30s-chair-stand test [45]. This test consists of counting the number of stand-sit on a chair cycles within $30 \mathrm{~s}$. Medication use, including medication for high blood pressure, for high cholesterol, insulin, metformin, and other medications for diabetes treatment, were self-reported by participants at baseline and checked against medical records.

\section{Statistical analysis}

Participants were classified in three categories (tertiles), depending on the inactive time in hours accumulated in one day. Tertile 1 (T1) included those participants accumulating less than $7.6 \mathrm{~h} /$ day of inactive time (low time spent with inactive behaviours). Tertile 2 (T2) included those participants who spent between 7.6 and $9.3 \mathrm{~h} /$ day inactive (moderate time spent with inactive behaviours). And tertile 3 (T3) included those participants accumulating between 9.3 and $15.1 \mathrm{~h}$ /day inactive (high time spent with inactive behaviours).

Descriptive characteristics were summarized as means and standard deviations (SDs) or as numbers and percentages (\%). One-way analysis of variance (ANOVA) and Chi-square tests $\left(\chi^{2}\right)$ were used to assess differences across tertiles of inactive time in hours/day for continuous and categorical variables respectively.

First generalized additive models were applied to ascertain about the linearity in the association between our exposures and outcomes. Given that there was no evidence of departure from linearly, multivariate linear regression analyses were used to estimate the $\beta$-coefficients and 95\% confidence intervals (CIs) for the associations between inactive time (continuous variable: bouts of $30 \mathrm{~min}$; categorical variable: sex-specific tertiles) and BMI, WC, body fat, body muscle mass, VAT, HbA1c, glucose, HDL, LDL, triglycerides, SBP and DBP. Our models were adjusted by the minimally sufficient adjustment set of covariables, determined using Directed Acyclic Graphs (DAGs) implemented in DAGitty software [46] available free on www. dagitty.net. The DAGs were built by identifying all known factors related to inactive time or our outcomes. Therefore our main models were adjusted for age, sex, educational level, marital status, erMedDiet, MVPA and smoking.

Logistic regression models were used to assess the association between categories of inactive time (tertiles) and the prevalence of the MetS's components, as well as prevalence of obesity and T2D. Prevalence ratios (PR) were calculated using the odds ratios (OR) obtained with logistic regression model. PR permits to assess the true ratios of prevalence in this sample, given the high prevalence of MetS, obesity and T2D in the present population, to avoid an overestimation of the risk. PR were calculated as $[(1-\mathrm{P0})+(\mathrm{PO} * \mathrm{OR})](\mathrm{PO}$ is the prevalence in the reference category) [47].

Linear regression modelling using an isotemporal substitution was used to quantify the associations of replacing $30 \mathrm{~min}$ of inactive time for $30 \mathrm{~min}$ of time in bed, LPA or MVPA on cardio-metabolic risk markers. Isotemporal substitution has been recommended for use in observational research using time-based measures of physical activity [30]. Prior to running the models, all activity patterns (time in bed, inactive time, LPA and MVPA) were divided by a constant of 30 , which was considered as an unit of time equivalent to $30 \mathrm{~min}$ (according to the PA guidelines [21, 22, 48]). Consequently, every unit increase represents exchanges of $30 \mathrm{~min}$ per day of any of these behaviours. To perform the 
isotemporal substitution models, a variable representing the total accelerometer wear time was constructed by adding up time in bed, inactive time, LPA and MVPA. This variable of wear time was entered simultaneously in the analysis with time in bed, LPA and MVPA. The resulting regression coefficient represents the association of re-allocating a unit of inactive time to a unit of time in bed, LPA and MVPA. Finally, the model was adjusted for age, sex, educational level, marital status, erMedDiet and smoking. Analyses follow published guideline for isotemporal substitution [31].

Sensitivity analyses were also conducted. Multiple adjusted linear and logistic regression models were adjusted for LPA, instead of MVPA, given that LPA is the most prevalent type of PA in our population and in older adults in general, and some studies had found beneficial effects of LPA on health $[4,5,26,27,49]$. In addition, multiple adjusted linear regression models were further adjusted for WC when assessing as an outcome: HbA1c, glucose, HDL, LDL, triglycerides, SBP and DBP. Finally, linear regression models shown in Table 2 were also adjusted for wear time, and results were consistent (data not shown).

In order to test whether the results of the isotemporal replacement models remained similar when using a proxy measure of sleep time, sleep time and time in bed were included in the analysis conjointly with the covariables mentioned above.

Statistical analyses were performed using Stata v15.0 program. $P$-values $<0.05$ were deemed as statistically significant. All analyses were conducted with data from database PREDIMED-Plus with date 2019-March-12.

\section{Results}

Table 1 presents a comparison of participants' characteristics among the three categories of inactive time. Participants in the upper tertile (T3) of inactivity were significantly older and had a higher BMI, WC, total body fat, VAT, HbA1c, glucose and triglycerides concentrations, and lower levels of total body muscle mass, and HDL cholesterol. Participants in T3 presented significantly higher prevalence of T2D (38\%) and obesity (78\%) compared to the other groups, and reported higher consumption of medication for the treatment of diabetes. Those in the most inactive category accumulated the least amount of time in bed, total PA, LPA and MVPA, and accumulated less repeats in the chair-stand test (all $p$ values $<0.001$ ). Lastly, the highest prevalence of smokers was found among those in T3 $(p<0.001)$.

Table 2 shows the $\beta$-coefficients (95\% CIs) for the associations between total inactive time, (both per 30-min bouts and in tertiles) and anthropometric measurements, body composition, biochemical parameters and blood pressure. Higher inactive time was associated with a worse adiposity and cardio-metabolic profile, including statistically significant higher BMI, WC, total body fat, VAT, HbAc1, glucose, triglycerides and DBP, and lower total body muscle mass and HDL cholesterol level.

Table 3 shows the prevalence ratios for obesity, T2D and the MetS's components by categories of inactive time. Those in the most inactive category (T3) showed significantly higher obesity prevalence ( $\mathrm{p}$ for trend = $0.014)$, significantly higher triglycerides $(p=0.005)$, and higher number of MetS components, $\geq 4$ components ( $\mathrm{p}$ for tend $=0.051$ ) and 5 components ( $\mathrm{p}$ for tend $=0.054$ ), compared to those with less inactive time (T1).

Table 4 shows the $\beta$-coefficients (95\% CIs) of the isotemporal substitution models. Figure 1 shows the same isotemporal substitution models but outcome variables had been standardized as z-scores to aid visualization of results. Isotemporal substitution of $30 \mathrm{~min}$ a day of inactive time with equivalent time in bed was associated with lower BMI, WC and HbA1c (all $p$-values $<0.05$ ); reallocating $30 \mathrm{~min}$ of inactive time per day with LPA or MVPA (i.e., decreasing inactive time at the expense of increasing LPA or MVPA time) was associated with lower BMI, WC, total body fat, VAT, HbA1c, glucose, triglycerides, and higher total body muscle mass and HDL (all p-values <0.05). Estimates of association were larger in all variables when replacing $30 \mathrm{~min}$ a day of inactive time by the equal amount of time in MVPA than when replacing it by LPA or time in bed.

No significant changes were observed when performing sensitivity analyses adjusting linear and logistic regression models for LPA instead of MVPA (See Additional file 1: Table S1 and Table S3), or for WC (See Additional file 1: Table S2). When running the isotemporal replacement models with sleep time instead of time in bed results also remained similar (See Additional file 1: Table S4).

\section{Discussion}

Results from this cross-sectional study show that time spent inactive was associated with a number of cardiometabolic risk factors in a sample of older adults, independent of PA levels. Overall, this study highlights that replacing $30 \mathrm{~min}$ a day of inactive time with an equal amount of MVPA, LPA and time in bed resulted in a significantly improved cardio-metabolic profile in men and women with the MetS.

The results from this study show that inactive time worsens the metabolic profile in an aging population with high cardio-metabolic risk, increasing the chances of cardiovascular events. This is similar to other studies where it has been found that high levels of inactivity, including sitting time, are associated with higher rates of obesity, triglycerides and MetS [50, 51] and premature mortality and diabetes [51,52] across different populations.

Isotemporal substitution analyses have public health implications $[4,9,13,23,25,28,29,31]$. Comparative 
Table 1 Baseline characteristics of the study population across categories of inactive time measured by accelerometer Tertiles of inactive time $(\mathrm{h} / \text { day })^{\mathrm{a}}$

\begin{tabular}{|c|c|c|c|c|c|c|}
\hline & Total $n$ & All & $\mathrm{T} 1 n=735$ & $\mathrm{~T} 2 n=729$ & $\mathrm{~T} 3 n=725$ & $P$ - value \\
\hline Age, years & 2189 & $65.0(4.95)$ & $64.1(4.77)$ & $65.2(4.94)$ & $65.8(4.98)$ & $<0.001$ \\
\hline Women, n (\%) & 2189 & $1032(47.1)$ & $345(46.9)$ & $343(47.1)$ & $344(47.5)$ & 0.979 \\
\hline \multicolumn{7}{|l|}{ Anthropometric measures } \\
\hline $\mathrm{BMI}\left(\mathrm{kg} / \mathrm{m}^{2}\right)$ & 2189 & $32.6(3.46)$ & $32.2(3.29)$ & $32.4(3.37)$ & $33.2(3.63)$ & $<0.001$ \\
\hline Waist circumference $(\mathrm{cm})$ & 2189 & $107(9.54)$ & $106(9.41)$ & $107(9.20)$ & 109 (9.78) & $<0.001$ \\
\hline \multicolumn{7}{|l|}{ DXA Body composition } \\
\hline Total body fat (\%) & 662 & $40.8(6.98)$ & $39.4(6.83)$ & $40.7(6.84)$ & $42.3(6.98)$ & $<0.001$ \\
\hline VAT (kg) & 651 & $2.27(0.90)$ & $2.12(0.85)$ & $2.32(0.90)$ & $2.38(0.93)$ & 0.006 \\
\hline Total body muscle mass (\%) & 662 & $56.1(6.62)$ & $57.4(6.50)$ & $56.2(6.50)$ & $54.7(6.61)$ & $<0.001$ \\
\hline \multicolumn{7}{|l|}{ Clinical parameters } \\
\hline $\mathrm{HbA1c}(\%)$ & 2009 & $6.15(0.87)$ & $6.08(0.81)$ & $6.12(0.79)$ & $6.26(0.99)$ & $<0.001$ \\
\hline Glucose (mg/dL) & 2164 & $114(29.5)$ & $113(27.9)$ & $113(27.8)$ & $117(32.4)$ & 0.035 \\
\hline $\mathrm{HDL}(\mathrm{mg} / \mathrm{dL})$ & 2173 & $47.8(11.9)$ & $49.1(12.0)$ & $47.7(11.9)$ & $46.6(11.6)$ & $<0.001$ \\
\hline $\mathrm{LDL}(\mathrm{mg} / \mathrm{dL})$ & 2127 & $121(46.5)$ & $122(31.9)$ & $122(51.0)$ & $119(53.7)$ & 0.459 \\
\hline Triglycerides (mg/dL) & 2178 & $152(78.9)$ & $141(74.9)$ & $151(71.4)$ & $165(87.7)$ & $<0.001$ \\
\hline $\mathrm{SBP}(\mathrm{mmHg})$ & 2175 & $140(17.4)$ & $140(17.0)$ & $141(17.3)$ & $140(18.0)$ & 0.293 \\
\hline $\mathrm{DBP}(\mathrm{mmHg})$ & 2175 & $80.3(10.1)$ & $80.6(9.88)$ & $80.0(9.92)$ & $80.2(10.4)$ & 0.430 \\
\hline Diabetes prevalence, n (\%) & 2189 & $749(34.2)$ & $229(31.2)$ & $246(33.7)$ & $274(37.8)$ & 0.027 \\
\hline Obesity prevalence, n (\%) & 2189 & $1610(73.6)$ & $519(70.6)$ & $528(72.4)$ & $563(77.7)$ & 0.007 \\
\hline \multicolumn{7}{|l|}{ Metabolic Syndrome } \\
\hline High blood pressure, n (\%) & 2189 & 1611 (73.6) & $534(72.7)$ & $542(74.3)$ & $535(73.8)$ & 0.755 \\
\hline High triglycerides, n (\%) & 2178 & 901 (41.4) & $244(33.4)$ & $305(42.0)$ & $352(48.9)$ & $<0.001$ \\
\hline Low HDL cholesterol, n (\%) & 2173 & $888(40.9)$ & $263(36.0)$ & $307(42.3)$ & $318(44.3)$ & 0.004 \\
\hline High glucose, n (\%) & 2189 & $1686(77.0)$ & $566(77.0)$ & $561(77.0)$ & $559(77.1)$ & 0.998 \\
\hline High waist circumference, n (\%) & 2189 & $2098(95.8)$ & $706(96.1)$ & $693(95.1)$ & $699(96.4)$ & 0.408 \\
\hline \multicolumn{7}{|l|}{ Physical activity behaviours ${ }^{\mathrm{b}}$} \\
\hline Time in bed (h/day) & 2189 & $8.06(1.29)$ & $8.28(1.25)$ & $8.23(1.21)$ & $7.68(1.33)$ & $<0.001$ \\
\hline Inactive time (h/day) & 2189 & $8.30(1.98)$ & $6.25(0.90)$ & $8.21(0.56)$ & $10.5(1.31)$ & $<0.001$ \\
\hline LPA (h/day) & 2189 & $2.54(1.07)$ & $3.45(0.99)$ & $2.45(0.74)$ & $1.72(0.63)$ & $<0.001$ \\
\hline MVPA (h/day) & 2187 & $0.67(0.54)$ & $0.93(0.59)$ & $0.66(0.49)$ & $0.43(0.40)$ & $<0.001$ \\
\hline Total PA (h/day) & 2187 & $3.22(1.33)$ & $4.38(1.22)$ & $3.11(0.84)$ & $2.15(0.78)$ & $<0.001$ \\
\hline Chair test 30s (repeats) & 2189 & $13.3(5.01)$ & $13.8(5.12)$ & $13.3(5.00)$ & $12.7(4.83)$ & $<0.001$ \\
\hline \multicolumn{7}{|l|}{ Sociodemographic/lifestyle data } \\
\hline Smoking habits, n (\%) & 2181 & & & & & $<0.001$ \\
\hline Never & & $942(43.2)$ & $328(44.8)$ & $329(45.4)$ & $285(39.4)$ & \\
\hline Current & & $251(11.5)$ & $61(8.3)$ & $75(10.3)$ & $115(15.9)$ & \\
\hline Former & & $988(45.3)$ & $343(46.9)$ & $321(44.3)$ & $324(44.7)$ & \\
\hline Educational level, n (\%) & 2171 & & & & & $<0.001$ \\
\hline Higher education/technician & & $474(21.8)$ & $113(15.5)$ & $176(24.3)$ & $185(25.8)$ & \\
\hline Secondary education & & $610(28.1)$ & $210(28.8)$ & $198(27.4)$ & $202(28.1)$ & \\
\hline Primary education/illiterate & & $1087(50.1)$ & $406(55.7)$ & $350(48.3)$ & $331(46.1)$ & \\
\hline Alcohol intake (g/day) & 2187 & $11.4(15.4)$ & $11.7(15.5)$ & $11.0(14.7)$ & $11.5(16.1)$ & 0.649 \\
\hline Energy intake (kcal/day) & 2187 & $2407(633)$ & $2444(640)$ & $2413(629)$ & $2364(628)$ & 0.051 \\
\hline
\end{tabular}


Table 1 Baseline characteristics of the study population across categories of inactive time measured by accelerometer (Continued)

\begin{tabular}{|c|c|c|c|c|c|c|}
\hline \multicolumn{7}{|l|}{ Tertiles of inactive time $(\mathrm{h} / \text { day })^{a}$} \\
\hline & Total $n$ & All & $\mathrm{T} 1 n=735$ & $\mathrm{~T} 2 n=729$ & $\mathrm{~T} 3 n=725$ & $P$-value \\
\hline MedDiet score (17 points) & 2189 & $8.56(2.70)$ & $8.64(2.75)$ & $8.61(2.63)$ & $8.42(2.71)$ & 0.243 \\
\hline Marital status & 2179 & & & & & 0.004 \\
\hline Not Married & & $546(25.1)$ & $161(22.0)$ & $174(23.9)$ & $211(29.3)$ & \\
\hline Married & & $1633(74.9)$ & $570(78.0)$ & $553(76.1)$ & $510(70.7)$ & \\
\hline \multicolumn{7}{|l|}{ Medication use for } \\
\hline High blood pressure & 2189 & $1700(77.7)$ & $554(75.4)$ & $563(77.2)$ & $583(80.4)$ & 0.065 \\
\hline Cholesterol & 2189 & $1159(53.0)$ & $378(51.4)$ & $384(52.7)$ & $397(54.8)$ & 0.437 \\
\hline Insulin & 2189 & $111(5.07)$ & $36(4.90)$ & $35(4.80)$ & $40(5.52)$ & 0.796 \\
\hline Metformin & 2189 & $544(24.9)$ & $160(21.8)$ & $184(25.2)$ & $200(27.6)$ & 0.035 \\
\hline Other medications for diabetes & 2189 & $514(23.5)$ & $151(20.5)$ & $171(23.5)$ & $192(26.5)$ & 0.028 \\
\hline \multicolumn{7}{|c|}{ 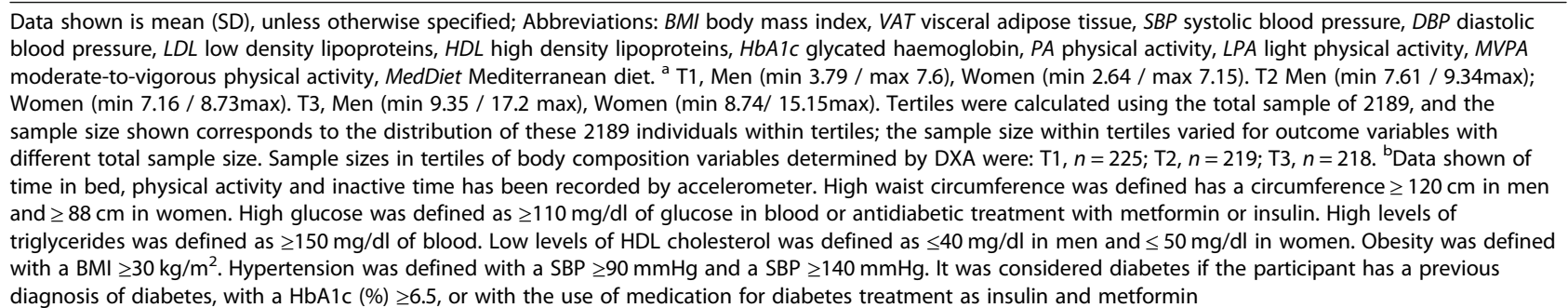 } \\
\hline
\end{tabular}

Table 2 Associations of total inactive time with adiposity indicators and cardio-metabolic risk factors

\begin{tabular}{|c|c|c|c|c|c|c|}
\hline \multirow[b]{2}{*}{ Outcome } & \multicolumn{3}{|c|}{ Tertiles of inactive time $(h / \text { day })^{a}$} & \multirow[t]{2}{*}{$p$ for trend } & \multirow{2}{*}{$\begin{array}{l}\text { Continuous } \\
\text { (per } 30 \mathrm{~min} / \mathrm{d} \text { of } \\
\text { inactive time) }\end{array}$} & \multirow[t]{2}{*}{$p$-value } \\
\hline & $\mathrm{T} 1 n=735$ & $\mathrm{~T} 2 n=729$ & $\mathrm{~T} 3 n=725$ & & & \\
\hline \multicolumn{7}{|l|}{ Anthropometric measures } \\
\hline BMI $\left(\mathrm{kg} / \mathrm{m}^{2}\right)$ & 0 (ref.) & $0.22(-0.14 ; 0.58)$ & $0.90(0.52 ; 1.29)$ & $<0.001$ & $0.11(0.06 ; 0.15)$ & $<0.001$ \\
\hline Waist circumference (cm) & 0 (ref.) & $-0.05(-0.99 ; 0.89)$ & $1.97(0.96 ; 2.97)$ & $<0.001$ & $0.26(0.15 ; 0.37)$ & $<0.001$ \\
\hline \multicolumn{7}{|l|}{ Body Composition } \\
\hline Total body fat (\%) & 0 (ref.) & $0.69(-0.14 ; 1.53)$ & $1.41(0.50 ; 2.32)$ & 0.002 & $0.17(0.07 ; 0.27)$ & 0.001 \\
\hline VAT (Kg) & 0 (ref.) & $0.16(0.00 ; 0.31)$ & $0.23(0.06 ; 0.39)$ & 0.013 & $0.03(0.01 ; 0.05)$ & 0.002 \\
\hline Total body muscle mass (\%) & 0 (ref.) & $-0.66(-1.46 ; 0.14)$ & $-1.30(-2.17 ;-0.44)$ & 0.003 & $-0.16(-0.25 ;-0.06)$ & 0.002 \\
\hline \multicolumn{7}{|l|}{ Clinical Parameters } \\
\hline HbA1c (\%) & 0 (ref.) & $0.01(-0.09 ; 0.10)$ & $0.11(0.01 ; 0.21)$ & 0.032 & $0.02(0.01 ; 0.03)$ & $<0.001$ \\
\hline Glucose (mg/dL) & 0 (ref.) & $0.08(-3.06 ; 3.22)$ & $2.68(-0.68 ; 6.05)$ & 0.116 & $0.47(0.11 ; 0.83)$ & 0.010 \\
\hline $\mathrm{HDL}(\mathrm{mg} / \mathrm{dL})$ & 0 (ref.) & $-1.02(-2.19 ; 0.15)$ & $-1.64(-2.89 ;-0.39)$ & 0.010 & $-0.20(-0.34 ;-0.07)$ & 0.003 \\
\hline LDL (mg/dL) & 0 (ref.) & $1.16(-3.87 ; 6.19)$ & $-1.23(-6.63 ; 4.17)$ & 0.652 & $-0.19(-0.76 ; 0.39)$ & 0.526 \\
\hline Triglycerides (mg/dL) & 0 (ref.) & $8.58(0.25 ; 16.9)$ & $20.0(11.0 ; 28.9)$ & $<0.001$ & $2.15(1.20 ; 3.11)$ & $<0.001$ \\
\hline $\mathrm{SBP}(\mathrm{mmHg})$ & 0 (ref.) & $1.43(-0.41 ; 3.27)$ & $0.34(-1.63 ; 2.32)$ & 0.740 & $-0.00(-0.21 ; 0.21)$ & 0.981 \\
\hline $\mathrm{DBP}(\mathrm{mmHg})$ & 0 (ref.) & $0.02(-1.03 ; 1.06)$ & $0.74(-0.38 ; 1.86)$ & 0.195 & $0.14(0.02 ; 0.26)$ & 0.022 \\
\hline
\end{tabular}

Values shown are $\beta(95 \% \mathrm{Cl})$. Abbreviations: BMI body mass index, VAT visceral adipose tissue, HbA1c glycated haemoglobin, $H D L$ high density lipoprotein, $L D L$ low density lipoprotein, SBP systolic blood pressure, DBP; diastolic blood pressure. Linear regression models were used to assess the association between inactive time and each cardiometabolic risk outcomes, adjusting for age, sex, education level, marital status, erMedDiet, moderate-vigorous physical activity and smoking. ${ }^{a}$ T1, Men ( $\min 3.79$ / $\max 7.6)$, Women $(\min 2.64$ / $\max 7.15)$. T2 Men $(\min 7.61$ / 9.34max); Women ( $\min 7.16$ / 8.73max). T3, Men $(\min 9.35$ / 17.2 $\max )$, Women $(\min$ $8.74 / 15.15 \mathrm{max}$ ). Tertiles were calculated using the total sample of 2189 , and the sample size shown corresponds to the distribution of these 2189 individuals within tertiles; the sample size within tertiles varied for outcome variables with different total sample size. Sample sizes in tertiles of body composition variables determined by DXA were: $\mathrm{T} 1, n=225 ; \mathrm{T} 2, n=219 ; \mathrm{T} 3, n=218$ 
Table 3 Prevalence Ratio of clinical and metabolic syndrome parameters according to tertiles of inactive time

\begin{tabular}{|c|c|c|c|c|}
\hline \multirow[b]{2}{*}{ Outcome } & \multicolumn{3}{|c|}{ Tertiles of inactive time $\left(\mathrm{h} /\right.$ day) ${ }^{a}$} & \multirow{2}{*}{$\begin{array}{l}p \text { for } \\
\text { trend }\end{array}$} \\
\hline & $\mathrm{T} 1 n=735$ & $\mathrm{~T} 2 n=729$ & $\mathrm{~T} 3 n=725$ & \\
\hline Obesity prevalence & 1 (ref.) & $1.02(0.95 ; 1.09)$ & $1.09(1.02 ; 1.15)$ & 0.014 \\
\hline Diabetes prevalence & 1 (ref.) & $1.05(0.89 ; 1.21)$ & $1.14(0.96 ; 1.32)$ & 0.125 \\
\hline \multicolumn{5}{|l|}{ Metabolic Syndrome Components } \\
\hline High blood pressure & 1 (ref.) & $0.97(0.93 ; 1.00)$ & $1.01(0.98 ; 1.03)$ & 0.580 \\
\hline High triglycerides & 1 (ref.) & $1.11(1.01 ; 1.21)$ & $1.16(1.05 ; 1.26)$ & 0.005 \\
\hline Low HDL cholesterol & 1 (ref.) & $1.08(0.96 ; 1.22)$ & $1.05(0.90 ; 1.19)$ & 0.533 \\
\hline High glucose & 1 (ref.) & $0.99(0.93 ; 1.05)$ & $0.99(0.92 ; 1.05)$ & 0.674 \\
\hline High waist circumference & 1 (ref.) & $0.99(0.96 ; 1.01)$ & $1.00(0.97 ; 1.02)$ & 0.954 \\
\hline$\geq 4$ components metabolic syndrome & 1 (ref.) & $1.09(0.97 ; 1.21)$ & $1.13(1.00 ; 1.25)$ & 0.051 \\
\hline 5 components metabolic syndrome & 1 (ref.) & $1.08(0.82 ; 1.40)$ & $1.30(0.99 ; 1.67)$ & 0.054 \\
\hline
\end{tabular}

Values shown are prevalence ratios $(95 \% \mathrm{Cl}$ ). Abbreviations: HDL high-density lipoprotein. Logistic regression models were adjusted for age, sex, educational level, marital status, erMedDiet, moderate-vigorous physical activity and smoking status. Tertiles were calculated using the total sample of 2189, and the sample size shown corresponds to the distribution of these 2189 individuals within tertiles; the sample size within tertiles varied for outcome variables with different total sample size

${ }^{\mathrm{a}} \mathrm{T} 1$, Men $(\min 3.79$ / $\max 7.6)$, Women $(\min 2.64$ / $\max 7.15)$. T2 Men (min 7.61 / 9.34max); Women (min 7.16 / 8.73max). T3, Men (min 9.35 / 17.2 max), Women (min $8.74 / 15.15 \mathrm{max})$. Tertiles were calculated using the total sample of 2189 , and the sample size shown corresponds to the distribution of these 2189 individuals within tertiles.

Table 4 Isotemporal substitution of inactive time (30 min/day) with time in bed and physical activity on cardio-metabolic risk

\begin{tabular}{|c|c|c|c|c|c|c|}
\hline Outcome & Inactive time with time in bed & $p$-value & Inactive time with LPA & $p$-value & Inactive time with MVPA & $p$-value \\
\hline \multicolumn{7}{|l|}{ Anthropometric measures } \\
\hline BMI $\left(\mathrm{kg} / \mathrm{m}^{2}\right)$ & $-0.12(-0.18 ;-0.05)$ & $<0.001$ & $-0.19(-0.27 ;-0.11)$ & $<0.001$ & $-0.40(-0.55 ;-0.25)$ & $<0.001$ \\
\hline Waist circumference (cm) & $-0.29(-0.46 ;-0.13)$ & 0.001 & $-0.42(-0.62 ;-0.21)$ & $<0.001$ & $-1.11(-1.49 ;-0.72)$ & $<0.001$ \\
\hline \multicolumn{7}{|l|}{ Body composition } \\
\hline Total body fat (\%) & $-0.07(-0.21 ; 0.08)$ & 0.391 & $-0.43(-0.62 ;-0.25)$ & $<0.001$ & $-0.69(-1.03 ;-0.34)$ & $<0.001$ \\
\hline VAT (Kg) & $-0.03(-0.05 ; 0.00)$ & 0.052 & $-0.06(-0.09 ;-0.03)$ & 0.001 & $-0.06(-0.12 ; 0.01)$ & 0.075 \\
\hline Total body muscle mass (\%) & $0.05(-0.09 ; 0.20)$ & 0.465 & $0.40(0.22 ; 0.56)$ & $<0.001$ & $0.62(0.29 ; 0.95)$ & $<0.001$ \\
\hline \multicolumn{7}{|l|}{ Clinical parameters } \\
\hline HbA1c (\%) & $-0.02(-0.04 ;-0.01)$ & 0.006 & $-0.03(-0.05 ;-0.01)$ & 0.002 & $-0.08(-0.12 ;-0.05)$ & $<0.001$ \\
\hline Glucose (mg/dL) & $-0.09(-0.64 ; 0.45)$ & 0.736 & $-1.05(-1.73 ;-0.38)$ & 0.002 & $-2.15(-3.44 ;-0.87)$ & 0.001 \\
\hline $\mathrm{HDL}(\mathrm{mg} / \mathrm{dL})$ & $0.11(-0.10 ; 0.31)$ & 0.306 & $0.27(0.01 ; 0.52)$ & 0.039 & $1.15(0.67 ; 1.63)$ & $<0.001$ \\
\hline LDL (mg/dL) & $0.30(-0.58 ; 1.19)$ & 0.502 & $0.34(-0.75 ; 1.43)$ & 0.538 & $1.43(-0.63 ; 3.48)$ & 0.174 \\
\hline Triglycerides (mg/dL) & $-0.89(-2.35 ; 0.58)$ & 0.235 & $-1.98(-3.78 ;-0.17)$ & 0.032 & $-9.40(-12.8 ;-5.99)$ & $<0.001$ \\
\hline $\mathrm{SBP}(\mathrm{mmHg})$ & $0.09(-0.24 ; 0.41)$ & 0.597 & $-0.02(-0.42 ; 0.38)$ & 0.906 & $0.35(-0.41 ; 1.11)$ & 0.363 \\
\hline $\mathrm{DBP}(\mathrm{mmHg})$ & $-0.07(-0.26 ; 0.11)$ & 0.431 & $-0.08(-0.30 ; 0.15)$ & 0.505 & $0.36(-0.07 ; 0.79)$ & 0.105 \\
\hline
\end{tabular}

Values shown are $\beta(95 \% \mathrm{Cl})$. These represent the change in outcome variables when substituting 30 min/day of inactive time with time in bed and physical activity. Abbreviations: LPA light physical activity, MVPA moderate-vigorous physical activity, BMI body mass index, VAT visceral adipose tissue, HbA1c glycated haemoglobin, $H D L$ high density lipoprotein, $L D L$ low-density lipoprotein, $S B P$; systolic blood pressure, $D B P$ diastolic blood pressure. Linear regression models were used to assess isotemporal substitution of inactive time with: time in bed, light PA and MVPA, adjusting for age, sex, educational level, marital status, erMedDiet, smoking and total wear time

${ }^{\mathrm{a}} \mathrm{T} 1$, Men $(\min 3.79$ / $\max 7.6)$, Women $(\min 2.64$ / $\max 7.15)$. T2 Men (min 7.61 / 9.34max); Women (min 7.16 / 8.73max). T3, Men (min 9.35 / 17.2 max), Women ( $\min 8.74 / 15.15 \mathrm{max})$. Tertiles were calculated using the total sample of 2189 , and the sample size shown corresponds to the distribution of these 2189 individuals within tertiles 


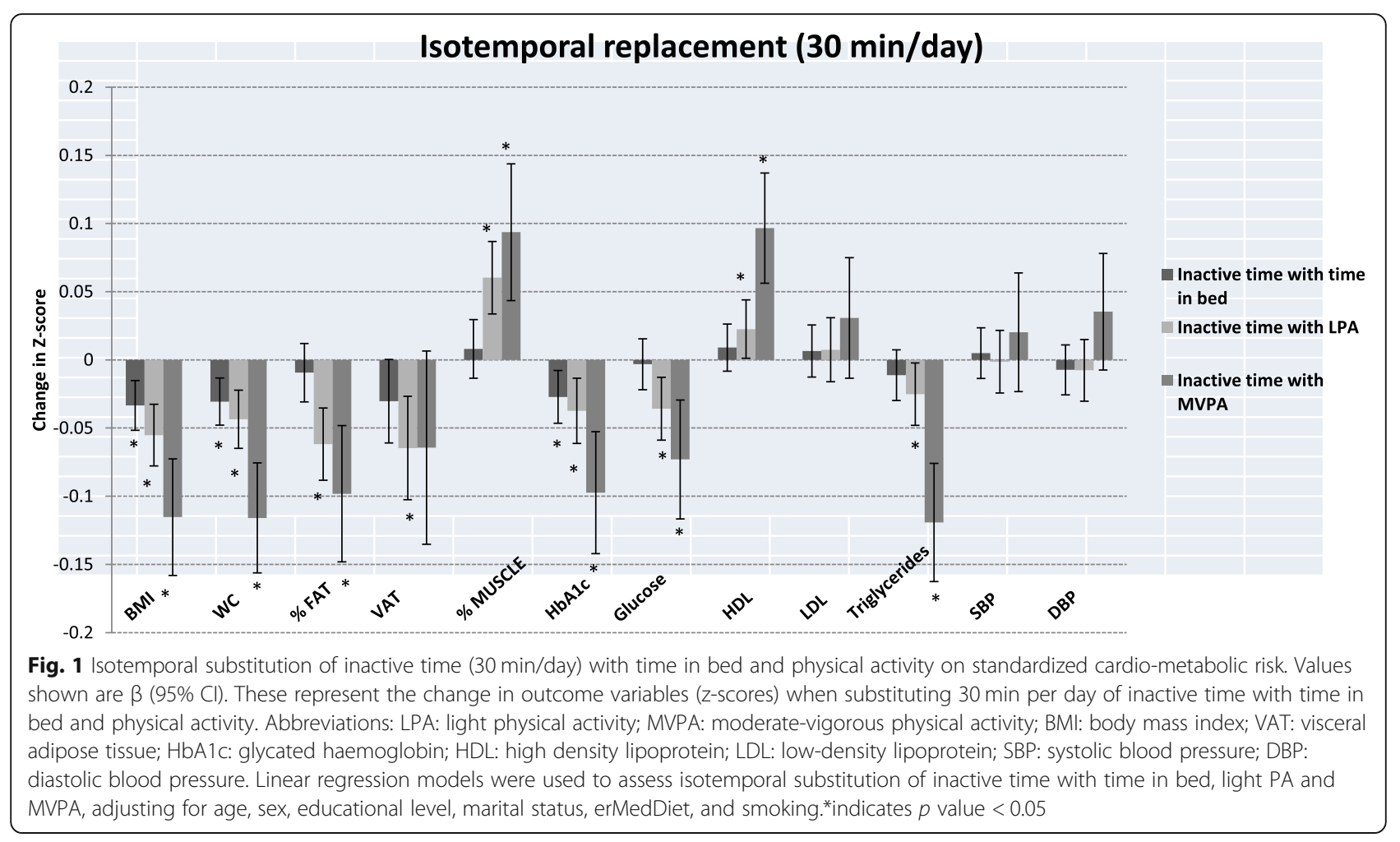

research in older adults is limited, especially in a population with chronic conditions, such as MetS [25]. Thus, the present results are in line with previous research conducted in adults (18-79 years) [4, 9, 13, 23, 25], which shows the beneficial effects of exchanging an unit of time spent inactive with equal amounts of PA or sleep on cardio-metabolic risk factors, including obesity and lipid profile. This study shows that replacing inactive time with any other behaviors has beneficial effects on cardio-metabolic risk, and these benefits increase proportionally. For instance, replacing $30 \mathrm{~min} / \mathrm{d}$ of inactive time with $30 \mathrm{~min} / \mathrm{d}$ of time in bed was associated with a lower WC of $0.26 \mathrm{~cm}$, whereas replacing this amount of inactive time with LPA resulted in a WC of $-0.45 \mathrm{~cm}$ and with MVPA in a WC of $-1.08 \mathrm{~cm}$.

Previous studies in adults have highlighted the benefits of replacing inactive time with MVPA, with the greatest benefits on improved BMI [9], T2D [9, 23], triglycerides, HbA1c [23] and glucose. Similarly, this study shows that interchanging $30 \mathrm{~min}$ of time inactive by MVPA was significantly associated with improvements in BMI, WC, body fat, muscle mass, HbA1c, glucose, HDL and triglycerides. Furthermore, the present study shows that health benefits are also attained when time inactive is replaced by LPA or time in bed, with improvements on: BMI, WC, body fat, VAT, muscle mass, HbA1c, glucose, HDL and triglycerides (LPA); and BMI, WC and HbAlc (time in bed). This is of interest, as research on this area continuous to mount, however findings remain ambiguous $[4,9,10,13,23-25$, 23 and although there are some studies in adult population $[4,25,28,29]$, few research has been conducted in older adults $[23,26]$ and, as far as we know, none in individuals with overweight/obesity and metabolic syndrome.

Given the prevalence of MetS, and the prevalence of an population aging worldwide, effective and sustainable long term actions are needed. Understanding the beneficial effects of substituting time inactive with different activity levels and sleep in high risk and aging populations is of importance as it will help defining future tailored health interventions. Multicomponent interventions to increase PA and decrease inactive time, using a multidisciplinary approach are recommended. According to our results, the promotion of MVPA would be of most benefit, however in older adults designing health interventions focused on LPA and sleep might be more appropriate. Interventions focused on LPA and sleep might result more feasible, appealing and might help improve attrition and sustainability in the long term, as they will not need continuous supervision and are easy to implement at home or care homes.

A marked strength of this study was the use of a large cohort of older men and women, with overweight/obesity and MetS. It is important to highlight that only objective and validated measurements were used for this study for both exposure and outcome variables. This reduces any potential bias or measurement error and increases the opportunities for comparison across the literature. In terms 
of limitations, the cross-sectional design prevents the assessment of causality. Given that exposure and outcome variables were measured simultaneously, we cannot rule out reverse causation, i.e. our outcomes, such as obesity, may have preceded inactivity, and not the other way round. In addition, due to its cross-sectional study design, the isotemporal replacement model used in this study is not based on actual replacements of one activity for another and should be interpreted at the population level; longitudinal studies are necessary to confirm the results obtained in this study. Selection of older subjects with overweight/obesity and MetS for the study cohort limits extrapolation of findings to other populations, including younger, leaner or healthier subjects. Moreover, this study was limited to Caucasians, hence the associations found may not be applicable to other ethnic groups. Thus, replicating this research in different ethnic groups with different lifestyles and fat distribution would be of interest. Methodological limitations of differentiating between sitting, standing are also important to consider. The wristworn 3-axial accelerometers used in this study quantify time spent in different intensities of activity based on specific count thresholds. This method works reasonably well for identifying inactive, LPA and MVPA but it is limited in its capacity to distinguish between standing and reclining postures. Thus, throughout this paper we refer to "inactivity" (activities of < 1.5 METs during day-time) and not "sedentarism" (meaning activities of $<1.5 \mathrm{METs}$ /day in seated or reclining positions). Another limitation is the use of the cutoff intensity level points to cluster data as inactive, LPA or MVPA time. Cut points are normally population and protocol specific, limiting the possibility of comparison across studies and populations [35]. Finally, although we used validated algorithms to estimate time in bed from accelerometer data without the use of sleep diaries, sleep time estimates were less accurate, which prevented us from using sleep time in the main analyses. This issue has been overcame by using several sophisticated analysis to assess the complex inter-relationships between different lifestyle behaviours in relation to cardiometabolic risk factors.

\section{Conclusion}

These results add to the growing literature using Isotemporal Replacement methods and it is one of the few focused on older adults with the metabolic syndrome. Results from this cross-sectional study indicate that replacing inactive time with any PA and time in bed was associated with improved cardio-metabolic factors in older adults with overweight or obesity and the MetS. Our findings support the notion that PA and inactive time are both linked with health outcomes and that both behaviors should be included in public health guidelines. Future intervention studies are needed to confirm causality.
Tailored health intervention research with a focus on sleep, LPA and MVPA are recommended.

\section{Supplementary information}

Supplementary information accompanies this paper at https://doi.org/10. 1186/s12966-019-0892-4

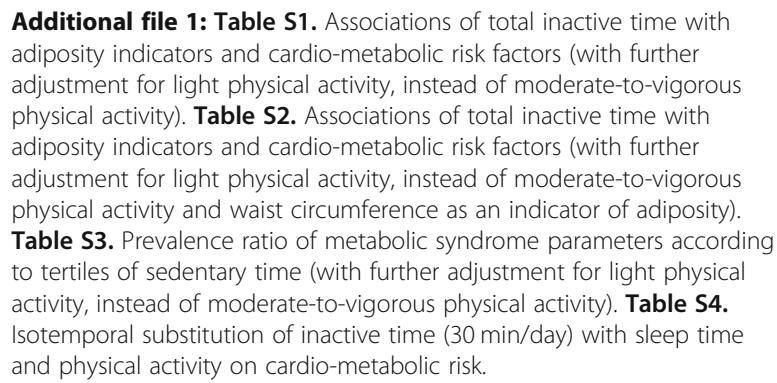

\section{Abbreviations}

BMI: Body mass index; Cls: Confidence intervals; DAG: Directed acyclic graphs; DBP: Diastolic blood pressure; DXA: Dual-energy X-ray absorptiometry; erMedDiet: Energy-restricted traditional Mediterranean Diet; HbA1c: Glycated hemoglobin; HDL: High-density lipoprotein cholesterol; LDL: Low-density lipoprotein cholesterol; LPA: Light physical activity; METs: Metabolic equivalent tasks; MetS: Metabolic syndrome; MVPA: Moderate-to-vigorous physical activity; OR: Odds ratios; PA: Physical activity; PR: Prevalence ratios; SBP: Systolic blood pressure; SDs: Standard deviations; T2D: Type 2 diabetes; VAT: Visceral adipose tissue; WC: Waist circumference; WHO: World health organization

\section{Acknowledgements}

The authors thank the participants for their enthusiastic collaboration, and the PREDIMED-Plus personnel and investigators, as well as all affiliated primary care centers, for their excellent work. Authors also thaks the IdISBa funding with the Liberi program for publishing in open acces journals.

\section{Authors' contributions}

AMGP and DR conceived of the study. AMGP and WM completed the statistical analysis. AMGP, WM and DR drafted the manuscript. DR supervised the study. All authors were involved in oversight of recruitment, data collection, revision of the manuscript and read and approved the final manuscript.

\section{Authors' information}

Not applicable.

\section{Funding}

The PREDIMED-Plus trial was supported by the official funding agency for biomedical research of the Spanish government, ISCIII through the Fondo de Investigación para la Salud (FIS), which is co-funded by the European Regional Development Fund (four coordinated FIS projects led by Jordi Salas-Salvadó and Josep Vidal, including the following projects: PI13/00673, PI13/00492, PI13/00272, PI13/01123, PI13/00462, PI13/00233, PI13/02184, PI13/00728, PI13/01090, PI13/01056, PI14/01722, PI14/00636, PI14/00618, PI14/00696, PI14/01206, PI14/01919, PI14/00853, PI14/01374, PI16/00473, PI16/00662, PI16/01873, PI16/01094, PI16/00501, PI16/00533, PI16/00381, PI16/00366, PI16/01522, PI16/01120, PI17/00764, PI17/01183, PI17/00855, PI17/01347, PI17/00525, PI17/01827, PI17/00532, PI17/00215, PI17/01441, PI17/00508, PI17/01732, PI17/00926); the Especial Action Project entitled: Implementación y evaluación de una intervención intensiva sobre la actividad física Cohorte PREDIMED-PLUS grant to Jordi Salas-Salvadó; the European Research Council grant (Advanced Research Grant 2013-2019; 340918) to Miguel Ángel Martínez-Gonzalez; the Recercaixa grant to Jordi Salas-Salvadó (2013ACUP00194); grants from the Consejería de Salud de la Junta de Andalucía (PI0458/2013; PS0358/2016; PI0137/2018); the PROMETEO/2017/017 grant from the Generalitat Valenciana; Grant of support to research groups no. 35/2011 (Balearic Islands Gov. \& FEDER funds) to 
Catalina M. Mascaró and Josep A. Tur; FPU Programme, PhD fellowship (Spanish Ministry of Science, Innovation \& Universities) to Catalina M. Mascaró; the Astra Zeneca Young Investigators Award in Category of Obesity and Diabetes 2017 to Dora Romaguera; Juan de la Cierva-formación research grant (FJCl-2015-24058) of the Spanish Ministry of Economy, Industry and Competitiveness and European Social Funds to Jadwiga Konieczna; the 'FOLIUM' programme within the FUTURMed project from the Fundación Instituto de Investigación Sanitaria Illes Balears (financed by 2017annual plan of the sustainable tourism tax and at 50\% with charge to the ESF Operational Program 2014-2020 ofthe Balearic Islands) to Jadwiga Konieczna. JR17/00022 contract to Olga Castaner from ISCIII. CIBERobn (Centros de Investigación Biomedica en Red: Obesidad y Nutrición), ClBEResp (Centros de Investigación Biomedica en Red: Epidemiología y Salud Publica) and CIBERdem (Centros de Investigación Biomedica en Red: Diabetes y Enfermedades). None of the funding sources took part in the design, collection, analysis or interpretation of the data and in writing the manuscript, or in the decision to submit the manuscript for publication.

\section{Availability of data and materials}

There are restrictions on the availability of data for the PREDIMED-Plus trial, due to the signed consent agreements around data sharing, which only allow access to external researchers for studies following the project purposes. Requestors wishing to access the PREDIMED-Plus trial data used in this study can make a request to the PREDIMED-Plus trial Steering Committee chair: jordi.salas@urv.cat. The request will then be passed to members of the PREDIMED-Plus Steering Committee for deliberation.

\section{Ethics approval and consent to participate}

Research Ethics Committees from all recruitment centers approved the study protocol, according to the ethical standards of the Declaration of Helsinki. All participants provided written informed consent. All centers have the ethics approval and consent from all the ethic committees. The trial was registered at the International Standard Randomized Controlled Trial (ISRCTN: http:// www.isrctn.com/ISRCTN89898870) with number 89898870 and registration date of 24 July 2014, retrospectively registered.

\section{Consent for publication}

\author{
Not applicable.
}

\section{Competing interests}

The authors declare that they have no competing interests.

\section{Author details}

'Centro de Investigación Biomédica en Red Fisiopatología de la Obesidad y la Nutrición (CIBEROBN), Institute of Health Carlos III, Madrid, Spain. ²Research Group on Nutritional Epidemiology \& Cardiovascular Physiopathology. Health Research Institute of the Balearic Islands (IdISBa), University Hospital Son Espases, Balearic Islands, Spain. ${ }^{3}$ School of Sport, Exercise and Health Science, Loughborough University, Loughborough, UK. ${ }^{4}$ School of Health Sciences, University of Málaga-Institute of Biomedical Research in Malaga (IBIMA), Málaga, Spain. ${ }^{5}$ Department of Preventive Medicine and Public Health, IDISNA, University of Navarra, Pamplona, Spain. ${ }^{6}$ Department of Nutrition, Harvard T.H. Chan School of Public Health, Boston, MA, USA. ${ }^{7}$ Universitat Rovira I Virgili, Departament de Bioquímica i Biotecnología, Unitat de Nutrició Humana, Reus, Spain. ${ }^{8}$ Institut d'Investigació Sanitària Pere Virgili (IISPV), Hospital Universitari Sant Joan de Reus, Unitat de Nutrició, Reus, Spain. ${ }^{9}$ Department of Preventive Medicine, University of Valencia, Valencia, Spain.

${ }^{10}$ Unit of Cardiovascular Risk and Nutrition, Institut Hospital del Mar d'Investigacions Mèdiques (IMIM), Barcelona, Spain. ${ }^{11}$ CIBER de Epidemiología y Salud Pública (CIBERESP), Instituto de Salud Carlos III, Madrid, Spain. ${ }^{12}$ Miguel Hernandez University, ISABIAL-FISABIO, Alicante, Spain. ${ }^{13}$ Bioaraba Health Research Institute; Osakidetza Basque Health Service, Araba University Hospital, University of the Basque Country UPV/EHU, Vitoria-Gasteiz, Spain.

${ }^{14}$ Department of Nutrition, Food Sciences, and Physiology, Center for Nutrition Research, University of Navarra, Pamplona, Spain. ${ }^{15}$ Precision Nutrition and Cardiometabolic Health program, IMDEA Food, CEI UAM + CSIC, Madrid, Spain. ${ }^{16}$ Nutrition Research Group, Research Institute of Biomedical and Health Sciences (IUIBS), University of Las Palmas de Gran Canaria, Las Palmas de Gran Canaria, Spain. ${ }^{17}$ Department of Internal Medicine, Institut d'Investigacions Biomèdiques August Pi Sunyer (IDIBAPS), Hospital Clinic, University of Barcelona, Barcelona, Spain. ${ }^{18}$ Virgen de la
Victoria Hospital, Department of Endocrinology, Instituto de Investigación Biomédica de Málaga (IBIMA), University of Málaga, Málaga, Spain.

${ }^{19}$ Department of Family Medicine, Research Unit, Distrito Sanitario Atención Primaria Sevilla, Sevilla, Spain. ${ }^{20}$ Lipids and Vascular Risk Unit, Internal Medicine, Hospital Universitario de Bellvitge, Hospitalet de Llobregat, Barcelona, Spain. ${ }^{21}$ Department of Medicine, Universidad de Barcelona, Barcelona, Spain. ${ }^{22}$ Research Group on Community Nutrition \& Oxidative Stress, University of Balearic Islands, Palma de Mallorca, Spain. ${ }^{23}$ Department of Internal Medicine, Maimonides Biomedical Research Institute of Cordoba (IMIBIC), Reina Sofia University Hospital, University of Cordoba, Cordoba, Spain. ${ }^{24}$ Department of Preventive Medicine and Public Health, University of Granada, Granada, Spain. ${ }^{25}$ Departamento de Ciencias de la Salud, Centro de Estudios Avanzados en Olivar y Aceites de Oliva, Universidad de Jaén, Jaén, Spain. ${ }^{26}$ Department of Endocrinology and Nutrition, Instituto de Investigación Sanitaria Hospital Clínico San Carlos (IdISSC), Madrid, Spain. ${ }^{27}$ Nutritional Genomics and Epigenomics Group, IMDEA Food, CEI UAM + CSIC, Madrid, Spain. ${ }^{28}$ Cátedrea Cronicidad Universidad Miguel Hernández. Fundación SEMERGEN, Elche, Spain. ${ }^{29}$ CIBER Diabetes y Enfermedades Metabólicas (CIBERDEM), Instituto de Salud Carlos III (ISCIII), Madrid, Spain. ${ }^{30}$ Department of Endocrinology, Institut d’ Investigacions Biomédiques August Pi Sunyer (IDIBAPS), Hospital Clinic, University of Barcelona, Barcelona, Spain. ${ }^{31}$ Department of Endocrinology and Nutrition, Hospital Fundación Jimenez Díaz Instituto de Investigaciones Biomédicas IISFJD, University Autonoma, Madrid, Spain. ${ }^{32}$ Lipid Clinic, Department of Endocrinology and Nutrition, Institut d'Investigacions Biomèdiques August Pi Sunyer (IDIBAPS), Hospital Clínic, Barcelona, Spain. ${ }^{33}$ Emergency Department, Complejo Hospitalario de Navarra, Servicio Navarro de Salud (Osasunbidea), Pamplona, Spain. ${ }^{34}$ Public Health Center Son Serra-La Vileta, Primary Care Management, Balearic Islands Health Service, Palma, Spain. ${ }^{35}$ Raval Public Health Center, Primary Care Management, Elche, Spain. ${ }^{36}$ Department of Clinical Health and Community Sciences (DISCCO), Università degli Studi di Milano, Milan, Italy.

Received: 15 July 2019 Accepted: 27 November 2019 Published online: 23 December 2019

\section{References}

1. Saklayen MG. The global epidemic of the metabolic syndrome. Curr Hypertens Rep. 2018;20:12.

2. Falconer $\mathrm{CL}$, Cooper AR, Walhin JP, Thompson D, Page AS, Peters TJ, et al. Sedentary time and markers of inflammation in people with newly diagnosed type 2 diabetes. Nutr Metab Cardiovasc Dis. 2014;24:956-62.

3. Chooi YC, Ding C, Magkos F. The epidemiology of obesity. Metabolism. 2019;92:6-10.

4. Colley RC, Michaud I, Garriguet D. Reallocating time between sleep, sedentary and active behaviours: associations with obesity and health in Canadian adults. Heal Reports. 2018;29:1-13.

5. Chastin SFM, Palarea-Albaladejo J, Dontje ML, Skelton DA. Combined effects of time spent in physical activity, sedentary behaviors and sleep on obesity and cardio-metabolic health markers: a novel compositional data analysis approach. Devaney J, editor. PLoS One. 2015;10:e0139984.

6. Breda J, Jewell J, Webber L, Galea G. WHO projections in adults to 2030. In: European congress on obesity; 2015. https://www.karger.com/Article/Pdf/3 82140. 02 May 2019.

7. Kelly T, Yang W, Chen C-S, Reynolds K, He J. Global burden of obesity in 2005 and projections to 2030. Int J Obes. 2008;32:1431-7.

8. Lin X, Zhang X, Guo J, Roberts CK, McKenzie S, Wu WC, et al. Effects of exercise training on cardiorespiratory fitness and biomarkers of cardiometabolic health: A systematic review and meta-analysis of randomized controlled trials. J Am Heart Assoc. 2015;4:1-28.

9. Rosique-Esteban N, Díaz-López A, Martínez-González MA, Corella D, Goday A, Martínez JA, et al. Leisure-time physical activity, sedentary behaviors, sleep, and cardiometabolic risk factors at baseline in the PREDIMED-PLUS intervention trial: a cross-sectional analysis. Song Y, editor. PLoS One. 2017; 12:e0172253.

10. Galmes-Panades AM, Konieczna J, Abete I, Colom A, Rosique-Esteban N, Zulet MA, et al. Lifestyle factors and visceral adipose tissue: results from the PREDIMED-PLUS study. PLoS One. 2019;14:1-15.

11. Ekelund U, Steene-Johannessen J, Brown WJ, Fagerland MW, Owen N, Powell KE, et al. Does physical activity attenuate, or even eliminate, the detrimental association of sitting time with mortality? A harmonised meta- 
analysis of data from more than 1 million men and women. Lancet. 2016; 388:1302-10.

12. Nagai K, Tamaki K, Kusunoki H, Wada Y, Tsuji S, Ito M, et al. Isotemporal substitution of sedentary time with physical activity and its associations with frailty status. Clin Interv Aging. 2018;13:1831-6.

13. Yates T, Henson J, Edwardson C, Dunstan D, Bodicoat DH, Davies MJ, et al. Objectively measured sedentary time and associations with insulin sensitivity: importance of reallocating sedentary time to physical activity. Prev Med. 2015;76:79-83.

14. Cooper AR, Sebire S, Montgomery AA, Peters TJ, Sharp DJ, Jackson N, et al. Sedentary time, breaks in sedentary time and metabolic variables in people with newly diagnosed type 2 diabetes. Diabetologia. 2012;55:589-99.

15. Same RV, Feldman DI, Shah N, Martin SS, Al Rifai M, Blaha MJ, et al. Relationship between sedentary behavior and cardiovascular risk. Curr Cardiol Rep. 2016:18:6

16. Hu FB, Leitzmann MF, Stampfer MJ, Colditz GA, Willett WC, Rimm EB. Physical activity and television watching in relation to risk for type 2 diabetes mellitus in men. Arch Intern Med. 2001;161:1542-8.

17. Hu FB, Li MY, Colditz GA, Willett WC, Manson JE. Television watching and other sedentary behaviors in relation to risk of obesity and type 2 diabetes mellitus in women. JAMA. 2003;289:1785.

18. Chau JY, Grunseit A, Midthjell K, Holmen J, Holmen TL, Bauman AE, et al. Cross-sectional associations of total sitting and leisure screen time with cardiometabolic risk in adults. Results from the HUNT Study, Norway. J Sci Med Sport. 2014;17:78-84.

19. Abete I, Konieczna J, Zulet MA, Galmés-Panades AM, Ibero-Baraibar I, Babio $\mathrm{N}$, et al. Association of lifestyle factors and inflammation with sarcopenic obesity: data from the PREDIMED-Plus trial. J Cachexia Sarcopenia Muscle. 2019. https://doi.org/10.1002/jcsm.12442.

20. Salas-Salvadó J, Díaz-López A, Ruiz-Canela M, Basora J, Fitó M, Corella D, et al. Effect of a lifestyle intervention program with energy-restricted Mediterranean diet and exercise on weight loss and cardiovascular risk factors: one-year results of the PREDIMED-plus trial. Diabetes Care. 2018;42: dc180836.

21. Murphy MH, Broom DR, Gill JMR, Gray CM, Jones A, Steele J, et al. UK physical activity guidelines: draft review and recommendations for adults (aged 19-64 years). 2018. https:/www.bristol.ac.uk/media-library/sites/sps/ documents/cmo/adults-technical-report.pdf. 30 April 2019.

22. Piercy KL, Troiano RP, Ballard RM, Carlson SA, Fulton JE, Galuska DA, et al. The physical activity guidelines for Americans. JAMA. 2018:9762:1-9.

23. Hamer M, Stamatakis E, Steptoe A. Effects of substituting sedentary time with physical activity on metabolic risk. Med Sci Sports Exerc. 2014;46:1946-50.

24. Maher CA, Mire E, Harrington DM, Staiano AE, Katzmarzyk PT. The independent and combined associations of physical activity and sedentary behavior with obesity in adults: NHANES 2003-06. Obesity. 2013;21:E730-7.

25. Buman MP, Winkler EAH, Kurka JM, Hekler EB, Baldwin CM, Owen N, et al. Reallocating time to sleep, sedentary behaviors, or active behaviors: associations with cardiovascular disease risk biomarkers, NHANES 20052006. Am J Epidemiol. 2014:179:323-34.

26. Lerma NL, Cho CC, Swartz AM, Miller NE, Keenan KG, Strath SJ. Isotemporal substitution of sedentary behavior and physical activity on function. Med Sci Sports Exerc. 2018;50:792-800.

27. Falconer CL, Page AS, Andrews RC, Cooper AR. The potential impact of displacing sedentary time in adults with type 2 diabetes. Med Sci Sports Exerc. 2015;47:2070-5.

28. Biddle G, Edwardson C, Henson J, Davies M, Khunti K, Rowlands A, et al. Associations of physical behaviours and behavioural reallocations with markers of metabolic health: a compositional data analysis. Int J Environ Res Public Health. 2018;15:2280.

29. Edwardson CL, Henson J, Bodicoat DH, Bakrania K, Khunti K, Davies MJ, et al. Associations of reallocating sitting time into standing or stepping with glucose, insulin and insulin sensitivity: a cross-sectional analysis of adults at risk of type 2 diabetes. BMJ Open. 2017;7:e014267.

30. Mekary RA, Willett WC, Hu FB, Ding EL. Isotemporal substitution paradigm for physical activity epidemiology and weight change. Am J Epidemiol. 2009:170:519-27.

31. Dumuid D, Pedišić Ž, Stanford TE, Martín-Fernández J-A, Hron K, Maher CA et al. The compositional isotemporal substitution model: a method for estimating changes in a health outcome for reallocation of time between sleep, physical activity and sedentary behaviour. Stat Methods Med Res. 2019;28:846-57.
32. Martínez-González MA, Buil-Cosiales P, Corella D, Bulló M, Fitó M, Vioque J, et al. Cohort profile: design and methods of the PREDIMED-Plus randomized trial. Int J Epidemiol. 2018:1-17.

33. Alberti KGMM, Eckel RH, Grundy SM, Zimmet PZ, Cleeman Jl, Donato KA, et al. Harmonizing the metabolic syndrome. Circulation. 2009;120:1640-5.

34. Brazendale K, Beets MW, Bornstein DB, Moore JB, Pate RR, Weaver RG, et al. Equating accelerometer estimates among youth: the Rosetta stone 2. J Sci Med Sport. 2016;19:242-9.

35. Rowlands AV, Edwardson CL, Davies MJ, Khunti K, Harrington DM, Yates T. Beyond cut points. Med Sci Sports Exerc. 2018;50:1323-32.

36. Herrmann SD, Barreira TV, Kang M, Ainsworth BE. How many hours are enough? Accelerometer wear time may provide bias in daily activity estimates. J Phys Act Health. 2013;10:742-9.

37. Rowlands AV, Mirkes EM, Yates T, Clemes S, Davies M, Khunti K, et al. Accelerometer-assessed physical activity in epidemiology. Med Sci Sports Exerc. 2018;50:257-65.

38. Healy GN, Matthews CE, Dunstan DW, Winkler EAH, Owen N. Sedentary time and cardio-metabolic biomarkers in US adults: NHANES 200306. Eur Heart J. 2011:32:590-7.

39. van Hees VT, Sabia S, Jones SE, Wood AR, Anderson KN, Kivimäki M, et al. Estimating sleep parameters using an accelerometer without sleep diary. Sci Rep. 2018:8:12975.

40. Hildebrand M, Van Hees VT, Hansen BH, Ekelund U. Age group comparability of raw accelerometer output from wrist- and hip-worn monitors. Med Sci Sports Exerc. 2014;46:1816-24.

41. van Hees VT, Sabia S, Anderson KN, Denton SJ, Oliver J, Catt M, et al. A novel, open access method to assess sleep duration using a wrist-worn accelerometer. PLoS One. 2015;10:e0142533.

42. van Hees VT, Fang Z, Langford J, Assah F, Mohammad A, da Silva ICM, et al. Autocalibration of accelerometer data for free-living physical activity assessment using local gravity and temperature: an evaluation on four continents. J Appl Physiol. 2014;1 17:738-44.

43. Kaul S, Rothney MP, Peters DM, Wacker WK, Davis CE, Shapiro MD, et al. Dual-energy $\mathrm{X}$-ray absorptiometry for quantification of visceral fat. Obesity (Silver Spring). 2012;20:1313-8.

44. Álvarez-Álvarez I, Martínez-González MÁ, Sánchez-Tainta A, Corella D, DíazLópez A, Fitó M, et al. Adherence to an energy-restricted Mediterranean diet score and prevalence of cardiovascular risk factors in the PREDIMEDplus: a cross-sectional study. Rev Esp Cardiol (English Ed). 2019;72:925-34.

45. Jones CJ, Rikli RE, Beam WC. A 30-s chair-stand test as a measure of lower body strength in community-residing older adults. Res Q Exerc Sport. 1999; 70:113-9.

46. Textor J, van der Zander B, Gilthorpe MS, Liśkiewicz M, Ellison GT. Robust causal inference using directed acyclic graphs: the R package "dagitty". Int J Epidemiol. 2016;45:1887-94.

47. Zhang J, Yu KF. What's the relative risk? JAMA. 1998:280:1690.

48. World Health Organization. World recommendations on physical activity for health. 2010. https://www.who.int/dietphysicalactivity/global-PA-recs-2010. pdf. 02 May 2019

49. Ekelund U, Tarp J, Steene-Johannessen J, Hansen BH, Jefferis B, Fagerland MW, et al. Dose-response associations between accelerometry measured physical activity and sedentary time and all cause mortality: systematic review and harmonised meta-analysis. BMJ. 2019. https://doi.org/10.1136/ bmj.14570.

50. Varela-Mato V, O'Shea O, King JA, Yates T, Stensel DJ, Biddle SJ, et al. Crosssectional surveillance study to phenotype lorry drivers' sedentary behaviours, physical activity and cardio-metabolic health. BMJ Open. 2017;7: e013162.

51. Wilmot EG, Edwardson CL, Achana FA, Davies MJ, Gorely T, Gray LJ, et al. Sedentary time in adults and the association with diabetes, cardiovascular disease and death: systematic review and meta-analysis. Diabetologia. 2012; 55:2895-905.

52. Edwardson CL, Gorely T, Davies MJ, Gray LJ, Khunti K, Wilmot EG, et al. Association of sedentary behaviour with metabolic syndrome: a metaanalysis. O'Connor KA, editor. PLoS One. 2012;7:e34916.

\section{Publisher's Note}

Springer Nature remains neutral with regard to jurisdictional claims in published maps and institutional affiliations. 